November 1, 2014 15:32 Powathil\&Chaplain Manuscript Powathil'BRL'R3

Biophysical Reviews and Letters

(C) World Scientific Publishing Company

\title{
Multiscale modelling of cancer progression and treatment control: The role of intracellular heterogeneities in chemotherapy treatment
}

\author{
Mark AJ Chaplain \\ Division of Mathematics, University of Dundee \\ Dundee, DD1 $4 H N$, Scotland \\ chaplain@maths.dundee.ac.uk \\ Gibin G Powathil \\ Division of Mathematics, University of Dundee \\ Dundee, DD1 $4 \mathrm{HN}$, Scotland \\ gibin@maths.dundee.ac.uk \\ Received Day Month Year \\ Revised Day Month Year
}

\begin{abstract}
Cancer is a complex, multiscale process involving interactions at intracellular, intercellular and tissue scales that are in turn susceptible to microenvironmental changes. Each individual cancer cell within a cancer cell mass is unique, with its own internal cellular pathways and biochemical interactions. These interactions contribute to the functional changes at the cellular and tissue scale, creating a heterogenous cancer cell population. Anticancer drugs are effective in controlling cancer growth by inflicting damage to various target molecules and thereby triggering multiple cellular and intracellular pathways, leading to cell death or cell-cycle arrest. One of the major impediments in the chemotherapy treatment of cancer is drug resistance driven by multiple mechanisms, including multi-drug and cell-cycle mediated resistance to chemotherapy drugs. In this article, we discuss two hybrid multiscale modelling approaches, incorporating multiple interactions involved in the sub-cellular, cellular and microenvironmental levels to study the effects of cell-cycle, phase-specific chemotherapy on the growth and progression of cancer cells.
\end{abstract}

Keywords: Multiscale modelling, cell-cycle pathway, hypoxia, chemotherapy

\section{Introduction}

Even with many important clinical and technological advancements in detecting and treating cancer, cure and control of many forms of cancer remain the greatest challenge to clinicians and scientists. In most cases, chemotherapy is used alone or in combination with other anticancer treatments such as radiotherapy and surgery to control a growing tumour. However, drug resistance driven by multiple mechanisms, including multi-drug and cell-cycle mediated resistances to chemotherapy drugs continues to be a major barrier for the treatment failure in human malignancies ${ }^{1,2}$. Several recent experimental studies have indicated the fundamental role of intratumoural heterogeneity as a driving source for the resistance to multiple chemother- 
November 1, 2014 15:32 Powathil\&Chaplain Manuscript Powathil'BRL'R3

2 MAJ Chaplain and GG Powathil

apeutic drugs ${ }^{3-4}$. One of the major reasons for this intratumoural heterogeneity is the intracellular perturbations in biochemical kinetics and heterogeneity in the tumour microenvironment that seriously impair the drug efficacy ${ }^{1}$. Hence, understanding various mechanisms involved in the development of drug resistance and, devising drugs and protocols to target these mechanisms are significant steps in overcoming drug resistance, where clinically driven computational models can play an important role ${ }^{5-6}$.

The two main factors that contribute to the intra-tumoural heterogeneity are internal cell-cycle dynamics and the surrounding oxygen concentration. The cell-cycle mechanism through which the cells duplicate consists of several transition phases of varying lengths and check points and is mainly divided into four phases. As most of the chemotherapeutic drugs that are administered to treat human malignancies are cell-cycle phase specific, they spare some of the cells that are in the non-targeted phase, causing a cell-cycle mediated drug resistance ${ }^{2}$. Cell-cycle dynamics are also further influenced by the external microenvironmental conditions, especially the availability of oxygen. Experimental evidence shows that hypoxia (lack of oxygen) can upregulate the expressions of some of the cyclin dependent kinase inhibitors such as p21 and p27, resulting in a prolonged cell-cycle time or even cell-cycle arrest ${ }^{7,8}$. This further contributes to the cell-cycle heterogeneity and cell-cycle phase specific drug resistance. Here, we discuss a multiscale mathematical model, incorporating some of these cellular heterogeneities to understand and study their role in inducing chemotherapeutic drug resistance.

The multiscale complexity of cancer progression warrants a multiscale modelling approach to produce truly clinically useful and predictive mathematical models. Previously, Powathil et al. ${ }^{5}$ developed a multiscale mathematical model of chemotherapy treatment, incorporating cell-cycle mediated intracellular heterogeneity and external oxygen heterogeneity to study the effects of cell-cycle, phase-specific chemotherapy and its combination with radiation therapy ${ }^{9}$. It has been shown that an appropriate combination of cell-cycle specific chemotherapeutic drugs with radiation delivery could effectively be used to control tumour progression. There have been several mathematical and computational modelling approaches developed to study the occurrence of drug resistance ${ }^{10-11}$. These approaches help to understand and to some extent analytically quantify various biological processes. Furthermore, it can also be used as a tool to analyse and design drug development experiments and clinical trials. In this article, we discuss the multiscale mathematical model developed by Powathil et al. ${ }^{5}$ and two different computational approaches to implement the developed model. Further, we use it to study the effects of cell-cycle phase-specific chemotherapeutic drugs on a growing tumour population with intratumoral heterogeneities ${ }^{6}$. 
November 1, 2014 15:32 Powathil\&Chaplain Manuscript Powathil'BRL'R3

Modelling of cancer progression and effects of chemotherapy 3

\section{Modelling Cancer Growth: Multiple Scales Involved}

Cancer growth is a complicated multiscale disease involving many interrelated processes that occur across a wide range of spatial and temporal scales, from the intracellular level to the tissue level. Consequently, a multiscale modelling approach is needed to capture the key processes that are occurring at these different spatial and temporal scales and couple them in an appropriate manner. Here we discuss a hybrid multiscale model developed by Powathil et al. ${ }^{5}$ that analyses the spatio-temporal dynamics at the level of individual cells, linking individual cell behaviour with the macroscopic behaviour of cell/tissue organisation and the microenvironment. The model captures the intracellular molecular dynamics of the cell-cycle pathway and the changes in oxygen dynamics within the tumour microenvironment. It is then used to study the the impact of oxygen heterogeneity on the spatio-temporal patterning of the cell distribution and their cell-cycle status ${ }^{5,9}$.

The growth and progression of a solid tumour mass depends critically on the responses of the individual cells that constitute the entire tumour mass. The evolution of each individual cancer cell and its decisions to grow, divide, remain inactive or die are usually influenced by the local micro-environmental conditions at the location occupied by any particular cell within the tumour and intracellular interactions, including the intracellular cell-cycle dynamics. Moreover, these cellular responses are actively influenced by various extracellular signals from neighbouring cells as well as its dynamically changing microenvironment. As discussed in Powathil et al. ${ }^{5}$, the growth and proliferation of each cancer cell is determined by its own internal cellcycle mechanism and is incorporated using a set of ordinary differential equations. This internal cell-cycle dynamics are further influenced by the changing surrounding oxygen concentration which is modelled through the activation of HIF pathway (hypoxia inducible factor pathway) linking the microenvironment to intracellular cell-cycle pathway.

The HIF pathway in usually implicated in several hypoxia related events within a growing tumour such as the production of metastatic phenotypes with increased mutation rates, increased secretion of angiogenic factors, less apoptosis and an upregulation of various pathways involved in the metastatic cascade ${ }^{12}$. The hypoxia inducible transcription factor -1 is composed of two subunits, HIF- $1 \alpha$ and HIF- $1 \beta$, both of which are required for its transcription activation function. Under normoxic conditions, the rapidly produced HIF- $1 \alpha$ is degraded immediately by the actions of proline hydroxylase and pVHL. However, under hypoxic conditions HIF-1 $\alpha$ escapes degradation and its level increases rapidly. This further activates the expression of various genes, triggering various intra- and intercellular pathways including the expressions of cyclin dependent kinase inhibitors p21 and p27 pathways, affecting the cell-cycle dynamics ${ }^{7,13}$. The dynamical changes in the tumour microenvironment due to the variations in oxygen concentration are modelled using partial differential equations. The developed model can be then used to analyse cellular heterogeneities due to various internal and external factors and its role in a cell's response to 
November 1, 2014 15:32 Powathil\&Chaplain Manuscript Powathil'BRL'R3

4 MAJ Chaplain and GG Powathil

chemotherapy treatment.

\subsection{Intracellular Heterogeneities: Modelling the Cell-Cycle Dynamics}

Most of the complex cellular processes that are involved in cancer progression such as proliferation, cell division and DNA replication are regulated by the cell-cycle. The cell-cycle is controlled by a complex hierarchy of metabolic and genetic networks with several transition phases of varying lengths and check points ${ }^{14}$. The cell-cycle can be divided into four main phases: S-phase where DNA synthesis occurs, G2-phase during which proteins required for mitosis are produced, M-phase where mitosis and separation occur and G1-phase where proteins necessary for Sphase progression are accumulated ${ }^{15}$. Additionally, cells may sometimes exit from the cell-cycle and enter a phase of quiescence or relative inactivity called the G0phase or resting phase ${ }^{14}$. The cell-cycle dynamics within a mammalian cell are regulated mainly by a family of cyclin dependent kinases (Cdk), whose activity is primarily dependent on association with a regulatory protein called cyclin ${ }^{16}$. Additionally, the progression of cell-cycle dynamics is affected by several intracellular and extracellular factors such as Cdk inhibitors that can act as negative regulators of the cell-cycle and tumour microenvironment ${ }^{15}$. A few specific examples of $\mathrm{Cdk}$ inhibitors include the proteins p16, p15, p21 and p27. Some of the extrinsic factors that can influence the cell-cycle mechanism include nutrient supply, cell size, temperature and cellular oxygen concentration ${ }^{14}$.

Here we use a cell-based modelling approach to study the growth and progression of a cancer cell mass. The evolution of each cancer cell is based on the decisions made by the cell-cycle mechanism within the cell and we further assume that this contributes to the intracellular heterogeneities. To model the cell-cycle dynamics within each cell, we use an adapted version of a very basic model ${ }^{5}$ originally developed by Tyson and Novak ${ }^{17,18}$ that includes only the interactions which are considered to be essential for cell-cycle regulation and control. The models by Tyson and Novak ${ }^{17,18}$ describe the cell-cycle as a hysteresis loop with two self-maintaining stages while the transitions between these two stages are determined by the changing cell mass during the division. They used kinetic relations between various chemical processes to study the transitions between two main steady states, G1 and S-G2-M of the cell-cycle, which is (in their model) controlled by changes in cell mass. Although, Tyson and Novak have subsequently introduced a much more sophisticated model for the mammalian cell-cycle ${ }^{19}$, for simplicity we have opted to use the six variable model to simulate the cell-cycle. Moreover in the adapted model, we have used the equivalent mammalian proteins stated in Tyson and Novak's paper, namely the Cdk-cyclin B complex [CycB], the APC-Cdh1 complex [Cdh1], the active form of the p55cdc-APC complex [p55 $\left.\mathrm{cdc}_{\mathrm{A}}\right]$, the total $\mathrm{p} 55 \mathrm{cdc}-\mathrm{APC}$ complex $\left[\mathrm{p} 55 \mathrm{cdc}_{\mathrm{T}}\right.$ ], the active form of Plk1 protein $[\mathrm{Plk} 1]$ and the mass of the cell $[\text { mass }]^{5,9}$. Using the kinetic relations, the evolution of the concentrations of these variables are mod- 
elled using the following system of six ODEs (further details concerning the kinetic interactions can be found in Tyson and Novak's papers ${ }^{17,18}$ ).

$$
\begin{aligned}
& \frac{d[\mathrm{CycB}]}{d t}=k_{1}-\left(k_{2}^{\prime}+k_{2}^{\prime \prime}[\mathrm{Cdh} 1]+[\mathrm{p} 27 / \mathrm{p} 21][\mathrm{HIF}]\right)[\mathrm{CycB}], \\
& \frac{d[\mathrm{Cdh} 1]}{d t}=\frac{\left(k_{3}^{\prime}+k_{3}^{\prime \prime}\left[\mathrm{p} 55 \mathrm{cdc}_{\mathrm{A}}\right]\right)(1-[\mathrm{Cdh} 1])}{J_{3}+1-[\mathrm{Cdh} 1]}-\frac{k_{4}[\mathrm{mass}][\mathrm{CycB}][\mathrm{Cdh} 1]}{J_{4}+[\mathrm{Cdh} 1]}, \\
& \frac{d\left[\mathrm{p} 55 \mathrm{cdc}_{\mathrm{T}}\right]}{d t}=k_{5}^{\prime}+k_{5}^{\prime \prime} \frac{([\mathrm{CycB}][\mathrm{mass}])^{n}}{J_{5}^{n}+\left([\mathrm{CycB}]\left[\mathrm{mass}^{\prime}\right]\right)^{n}}-k_{6}\left[\mathrm{p} 55 \mathrm{cdc}_{\mathrm{T}}\right], \\
& \frac{d\left[\mathrm{p} 55 \mathrm{cdc}_{\mathrm{A}}\right]}{d t}=\frac{k_{7}[\mathrm{Plk} 1]\left(\left[\mathrm{p} 55 \mathrm{cdc}_{\mathrm{T}}\right]-\left[\mathrm{p} 55 \mathrm{cdc}_{\mathrm{A}}\right]\right)}{J_{7}+\left[\mathrm{p} 55 \mathrm{cdc}_{\mathrm{T}}\right]-\left[\mathrm{p} 55 \mathrm{cdc}_{\mathrm{A}}\right]}-\frac{k_{8}[\mathrm{Mad}]\left[\mathrm{p}^{2} 5 \mathrm{cdc}_{\mathrm{A}}\right]}{J_{8}+\left[\mathrm{p} 55 \mathrm{cdc}_{\mathrm{A}}\right]} \\
& \frac{d[\mathrm{Plk} 1]}{d t}=k_{9}[\mathrm{mass}][\mathrm{CycB}](1-[\mathrm{Plk} 1])-k_{10}[\mathrm{Plk} 1], \\
& \frac{d[\mathrm{mass}]}{d t}=\mu[\mathrm{mass}]\left(1-\frac{\left[\mathrm{massc}_{\mathrm{A}}\right]}{m_{*}}\right),
\end{aligned}
$$

where $k_{i}$ are the rate constants and the values are chosen in proportion to those in Tyson and Novak so that the time scale is relevant to a mammalian cell-cycle ${ }^{5}$. Other parameters used in the system are $J_{i}$, [Mad] and $[\mathrm{p} 27 / \mathrm{p} 21]^{5}$. The effects of changes in oxygen dynamics are included into the system through the activation and inactivation of HIF pathway which further results in changes in cell-cycle length. Here, we have assumed that HIF-1 $\alpha$ concentration at a cellular position, which is normally inactive $([\mathrm{HIF}]=0)$, is activated $([\mathrm{HIF}]=1)$ if the oxygen concentration at that position falls below $10 \%$. The cell-cycle inhibitory effect of p21 or p27 genes expressed through the activation of HIF-1 $\alpha$ is incorporated into the equation governing our generic Cyclin-CDK dynamics, using an additional decay term proportional to the concentration of $\mathrm{p} 27 / \mathrm{p} 21$ (which is considered here as constant) ${ }^{5,20}$. A cell is assumed to divide when the concentration of Cdk-cyclin B complex [CycB] crosses a specific threshold value $[\mathrm{CycB}]_{t h}$ which is assumed to be 0.1 , from above, and then the mass, [mass] is halved. To introduce a random growth rate for individual cells which in turn introduces cell-cycle heterogeneity in the population, we consider a varying growth rate $\mu$. The rest of the parameter values of the cell-cycle model can be found in Powathil et al. ${ }^{5}$.

Figure 1 shows the changes in various protein concentrations that have been included in the current cell-cycle model for one single automaton cell. Every cell in this multiscale model has a similar cell-cycle dynamics built-in which further control the division and cell-cycle phases of the respective cells. In this representative figure (adapted from Powathil et al. ${ }^{5}$ ), a cell undergoes division constantly as long as there is enough space to divide and the surrounding microenvironment is favourable for its division. However, as soon as all its neighbouring spaces are occupied, the cell moves to a resting phase where the concentrations are maintained at a constant level. 


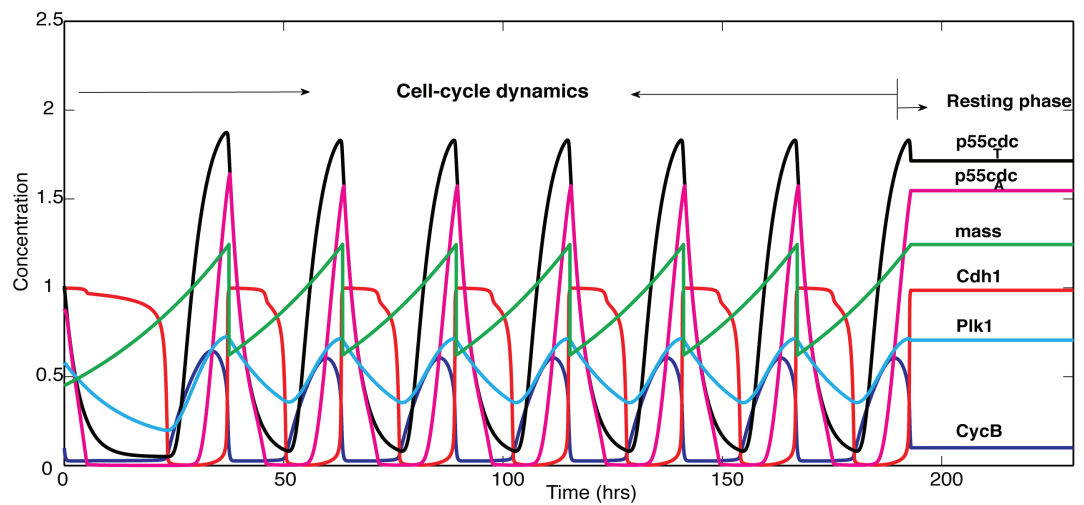

Fig. 1. Plot of the concentration profiles of the various intracellular proteins and the cell-mass over a period of 200 hours for one automaton cell in the model. This is obtained by solving the system of equations, (1) - (6), with the relevant parameter values. Adapted from Powathil et al. ${ }^{5}$.

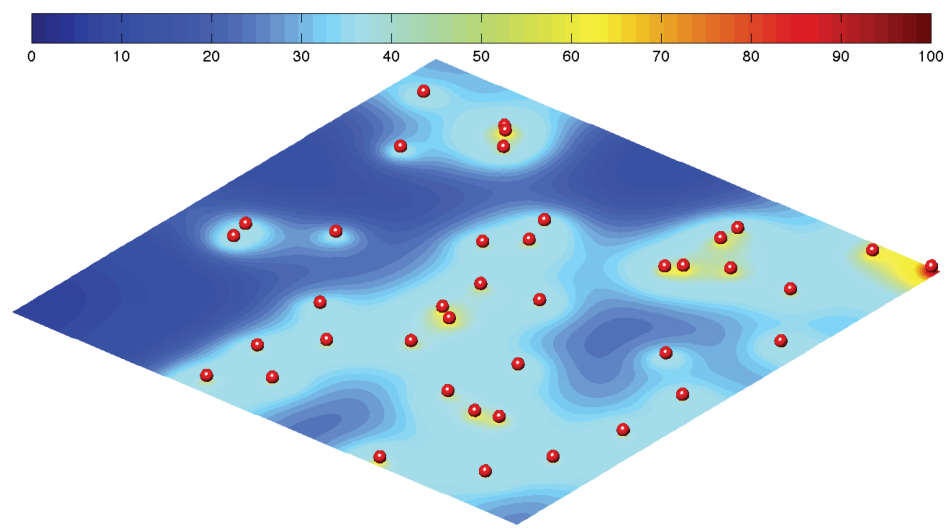

Fig. 2. Plot showing the concentration profile of oxygen supplied from the vasculature in the local tissue. The red coloured spheres represent the blood-vessel cross-sections and the colour map shows the percentages of oxygen concentration. Adapted from Powathil et al. ${ }^{9}$.

\subsection{Microenvironment Heterogeneities: Modelling the Oxygen Dynamics}

The growth of individual tumour cells as well as the entire tumour mass is externally influenced by its surrounding microenvironment. In particular the local availability of nutrients such as oxygen. The effects of a dynamically changing microenvironment introduced by incorporating oxygen dynamics, is modelled using a partial differential equation ${ }^{5,9}$. Here, oxygen is assumed to be supplied from a random distribution of blood vessels (vascular cross sections in 2D) with a density of $\phi_{d}=N_{v} / N^{2}$, where $N_{v}$ is the number of vessel cross sections in the 2-dimensional domain (of area $\left.N^{2}\right)^{5}$. This is a reasonable assumption if the blood vessels are as- 
November 1, 2014 15:32 Powathil\&Chaplain Manuscript $\quad$ Powathil'BRL R3

Modelling of cancer progression and effects of chemotherapy 7

sumed to be perpendicular to the tissue cross section of interest and there are no branching points through the plane of interest ${ }^{21,22}$. The temporal dynamics of these vessels are ignored at present, assuming the growth of tumour cells is much faster than that of the vessels within the time frame of interest. Denoting by $K(x, t)$ the oxygen concentration at position $x$ at time $t$, then its rate of change can be expressed as,

$$
\frac{\partial K(x, t)}{\partial t}=\nabla \cdot\left(D_{K}(x) \nabla K(x, t)\right)+r(x) m(x)-\phi K(x, t) \operatorname{cell}(x, t)
$$

where $D_{K}(x)$ is the diffusion coefficient and $\phi$ is the rate of oxygen consumption by a cell at position $x$ at time $t$ (cell $(x, t)=1$ if position $x$ is occupied by a cancer cell at time $t$ and zero otherwise). Here, $m(x)$ denotes the vessel cross section at position $x(m(x)=1$ for the presence of blood vessel at position $x$, and zero otherwise) and $r(x)$ describes rate of oxygen supply ${ }^{5}$. This equation is solved using no-flux boundary conditions and an initial condition ${ }^{23}$. Figure 2 shows a representative profile of the spatial distribution of oxygen concentration after solving equation (7) with relevant parameters ${ }^{5}$. Furthermore, It is observed that lack of an adequate supply of oxygen (hypoxia) can upregulate some of the cell-cycle inhibitory proteins such as p27 and p21 which could interfere with the cell-cycle, eventually taking the cell either to a resting phase or inducing a cell-cycle arrest ${ }^{24,7}$. These effects are introduced into the cell-cycle dynamics through the equation governing the changes of Cdk-cyclin B complex (cf. Equation 1).

\section{Implementation of the Multiscale Model}

The tissue-scale dynamics of the oxygen concentration outlined above can be linked to the sub-cellular and cellular changes through two different modelling approaches, namely, (i) a hybrid multiscale cellular automaton framework ${ }^{5,9}$ and (ii) a multiscale cellular Potts modelling approach using the CompuCell3D framework ${ }^{6}$. In both these modelling approaches, the computational domain contains three different components that are required to simulate the multiscale model. These are: (1) the cancer cells whose spatio-temporal evolution is controlled by internal cell-cycle dynamics and the external microenvironment; (2) the oxygen concentration distribution and (3) cross-sections of blood vessels from where the oxygen and chemotherapeutic drugs are supplied to the domain of interest. A detailed explanation of these modelling approaches are outlined below.

\subsection{The Hybrid Multiscale Cellular Automaton Framework}

Cellular automaton (CA) modelling has been used very extensively to model various aspects of tumour development and progression ${ }^{25,21,26,27}$. Some examples for such studies include multiscale tumour growth models by Alarcon et al. ${ }^{28}$, Ribba et al. ${ }^{29}$ and Gerlee and Anderson ${ }^{30}$. A brief review that discusses different CA modelling 


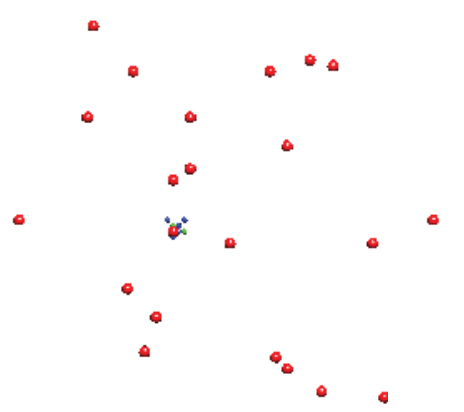

(a) Time $=100 \mathrm{hr}$

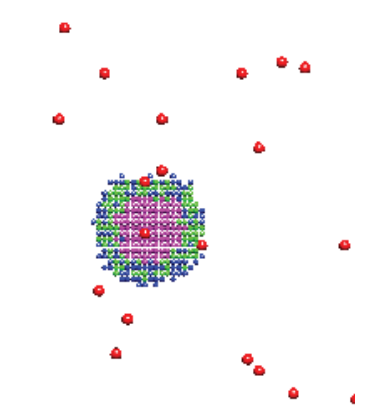

(b) Time $=300 \mathrm{hr}$

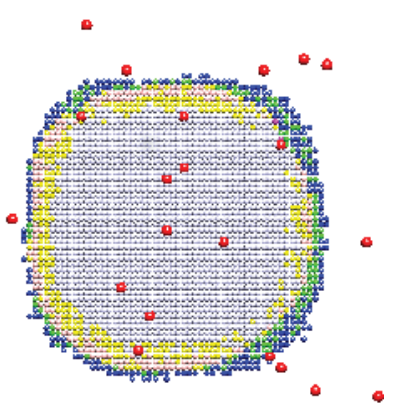

(c) Time $=600 \mathrm{hr}$

Fig. 3. Plots of the spatial distribution of the cells in different stages of the cell cycle which are G1 (blue), S-G2-M (green), resting (magenta), hypoxic cells in G1 (rose), hypoxic cells in S-G2-M (yellow) and hypoxic cells in resting (silver) at times 100, 300 and 600.

approaches to study various stages of cancer progression can be found in a review article by Moreira and Deutsch ${ }^{31}$. Recently, Powathil et al. ${ }^{5}$ developed a hybrid multiscale cellular automaton approach to model cancer progression and used the model to study the effects of cell-cycle dependent chemotherapeutic drugs alone and in combination with radiation therapy ${ }^{5,9}$.

The hybrid CA model is simulated on a spatial grid of size $100 \times 100$ grid points and each automaton element whether it is empty or occupied, has a physical size of $l \times l$, where $l=20 \mu \mathrm{m}$, simulating a cancer tissue of area $2 \times 2 \mathrm{~mm}^{2}$. The CA begins as a new grid of empty points with a single initial cell (a blue cell) at the centre of the grid in the G1-phase of the cell-cycle. This initial cell divides repeatedly following its internal cell-cycle dynamics and produces a cluster of cells on a square lattice (no-flux boundary conditions are imposed). The entire multiscale model is simulated over a certain period of time and a vector containing all cell positions and intracellular protein levels for each cell are updated accordingly. The oxygen dynamics are simulated using a finite difference scheme at every simulation time step and the corresponding oxygen concentration levels are updated. The cellcycle phases are determined using the concentration levels of [CycB]. If [CycB] is greater than a specific threshold (i.e. 0.1) the cell is considered to be in the S-G2-M phase (green cell) and if it is lower than this value, the cell is in the G1phase. If the cyclinB-cdk complex concentration [CycB] crosses this threshold from above, the cell undergoes cell division, its mass [mass] is halved. Alternatively, a cell may enter into a resting phase if the dividing cell's neighbourhood has no space for the new daughter cell. Alternatively, if division takes place, the new cell is placed into the G1-phase of the cell-cycle and is assigned a value for its proliferation rate $\mu$ randomly from the range of values of $\mu$. If there is more than one empty space with the same oxygen concentration level, a position is chosen randomly. The position of the new daughter cells is determined by alternating Moore and Von 


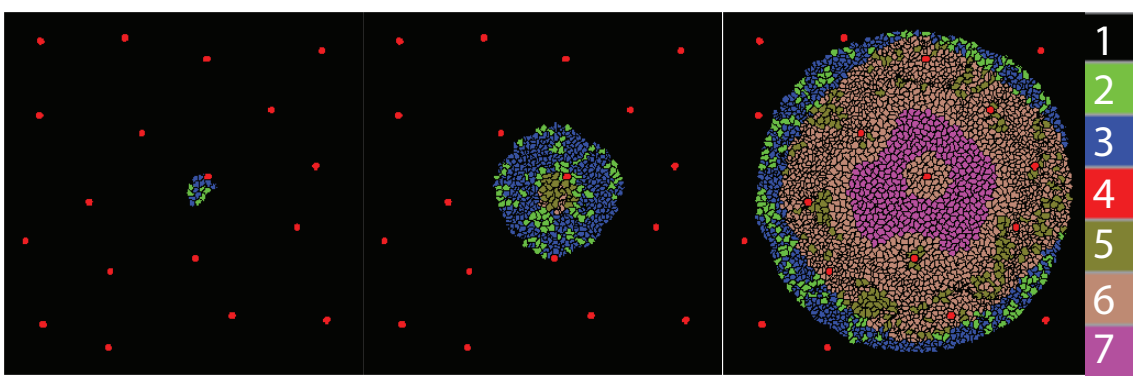

(a) Time $=150 \mathrm{hr}$

(b) Time $=350 \mathrm{hr}$

(c) Time $=650 \mathrm{hr}$

Fig. 4. Plots of the spatial evolution of tumour cells in different phases of the cell-cycle at times $=150 \mathrm{hr}, 350 \mathrm{hr}$ and $650 \mathrm{hr}$. The colour legend shows the types of the tumour cells; 1- medium, 2- G2 phase, 3- G1 phase, 4- vessel cross sections, 5- hypoxic G2 phase, 6- hypoxic G1 phase and 7- resting cells. Adapted from Powathil et al. ${ }^{6}$.

Neumann neighbourhoods to avoid generating cell distribution patterns matching the specific neighbourhood ${ }^{5,9}$ (this creates symmetric "circular" masses of cancer cells as opposed to square or diamond shapes). As the cancer cells proliferate, the oxygen demand increases making an imbalance between supply and demand which will eventually create a state where the cells are deprived of oxygen. If the oxygen concentration falls below $10 \%$ the cells are assumed to be hypoxic and the hypoxic cells that are in G1-phase are represented by rose colour coded cells while hypoxic S-G2-M cells and hypoxic resting cells are denoted by the colours yellow and silver, respectively. Figure 3 shows the distribution of cells in various cell-cycle phases at three different time points. The simulation time step for the both the CA model and the oxygen dynamics is taken as $\mathrm{T}=0.001 \mathrm{hr}$ as it gives a oxygen diffusion constant of $2 \times 10^{-5} \mathrm{~cm}^{2} / \mathrm{s}$ with appropriate diffusion length scale $L$ of $100 \mu \mathrm{m}$. Further details of the model can be found in Powathil et al. ${ }^{5}$.

\subsection{The Multiscale Cellular Potts Model: CompuCell3D Framework}

An alternative approach to modelling such complex multiscale problems is by using a multiscale cellular Potts model or the Glazier-Graner-Hogeweg (GGH) approach. The GGH model contains description of objects, such as cells and fields, interactions with the cellular properties that evolve with respect to time and space and are modelled with the help of various initial conditions ${ }^{32}$. Each cell is a collection of lattice pixels having the same index marker and is represented as spatially extended domains on a fixed lattice. We used the CompuCell3D framework developed by Glazier et al. (see http://www.compucell3d.org for details) to simulate the previously described multiscale model ${ }^{6}$. The multiscale model is simulated using a 2-dimensional lattice of size $300 \times 300$ pixels in the $x$ - and $y$-directions with an initial configuration of single cells surrounded by a number of blood vessel cross sections. Similar 
November 1, 2014 15:32 Powathil\&Chaplain Manuscript $\quad$ Powathil'BRL R3

to the previous approach, the division of tumour cells is driven by the cell-cycle dynamics modelled using the kinetic equations (1)-(5). The set of ODEs governing the cell-cycle dynamics is incorporated into the Compucell3D framework in each Monte Carlo time step (mcs) using Bionetsolver. Bionetsolver is a C++ library that permits easy definition of sophisticated models coupling reaction-kinetic equations described in SBML with the defined cells for execution in CompuCell3D ${ }^{32}$. Bionetsolver makes use of the SBML ODE Solver Library to implement reaction-kinetic network dynamics which can regulate the cell-cycle dynamics for each tumour cell within the domain. Cellular growth is incorporated into the model by incrementing the cell target volume in every mcs during growth phases at a constant rate of 0.5 times the current cell volume. Division is assumed to occur when the concentration of $[\mathrm{CycB}]$ crosses the threshold value from above. However, since here we are using a growing volume, the cell-cycle dynamics are simulated using the equations (1)-(5), using [volume] instead of [mass]. The parameter values of the cell-cycle model are scaled in such a way that each mcs step corresponds to 1 hour and hence a cell has an average cell-cycle length of 25-35 hours. The evolution of oxygen concentration is incorporated into the CompuCell3D as a diffusive chemical field that follows the respective PDE described previously. The parameters are taken from Powathil et al. ${ }^{5}$ and for consistency, the diffusion equation is simulated 1000 times in every 1 mcs to achieve a similar time-scale of $0.001 \mathrm{hr}{ }^{6}$. Figure 4 shows the spatial evolution of tumour cells. The colour of the tumour cells indicate their cell-cycle phase position and the microenvironment status.

\section{Modelling the Effects of Chemotherapy}

Chemotherapy is one of the most common therapeutic options for cancer treatment, either alone or in combination with other therapies (multimodality). Chemotherapeutic drugs act on rapidly proliferating cells targeting the different cell-cycle phases and check points. In cancer, Cdks, the proteins responsible for the activation of the cell-cycle, are over-expressed while cell-cycle inhibitory proteins are under-expressed which results in a malfunctioning in the regulation of the cell-cycle, and eventually leads to a promotion of uncontrolled growth. The rationale behind cell-cycle, phase-specific chemotherapy is to target those proteins that are overexpressed during various stages of cancer progression, inducing an inhibitory effect and thus controlling cell growth. One of the major issues that affects the delivery and effectiveness of chemotherapeutic drugs is the occurrence of cell-cycle mediated drug resistance ${ }^{2}$. This may be due to the presence of functionally heterogeneous cells and cell subpopulations, and can be addressed to some extent by using combinations of chemotherapy drugs that target different phases of the cell-cycle kinetics ${ }^{2}$.

We are interested in studying the effects of cell-cycle based chemotherapeutic drugs on cancer cells and cancer cell subpopulations with varying drug sensitivities. We model the spatio-temporal evolution of cell-cycle specific chemotherapeutic drugs using a similar partial differential equation as that governing the oxygen 
November 1, 2014 15:32 Powathil\&Chaplain Manuscript Powathil'BRL'R3

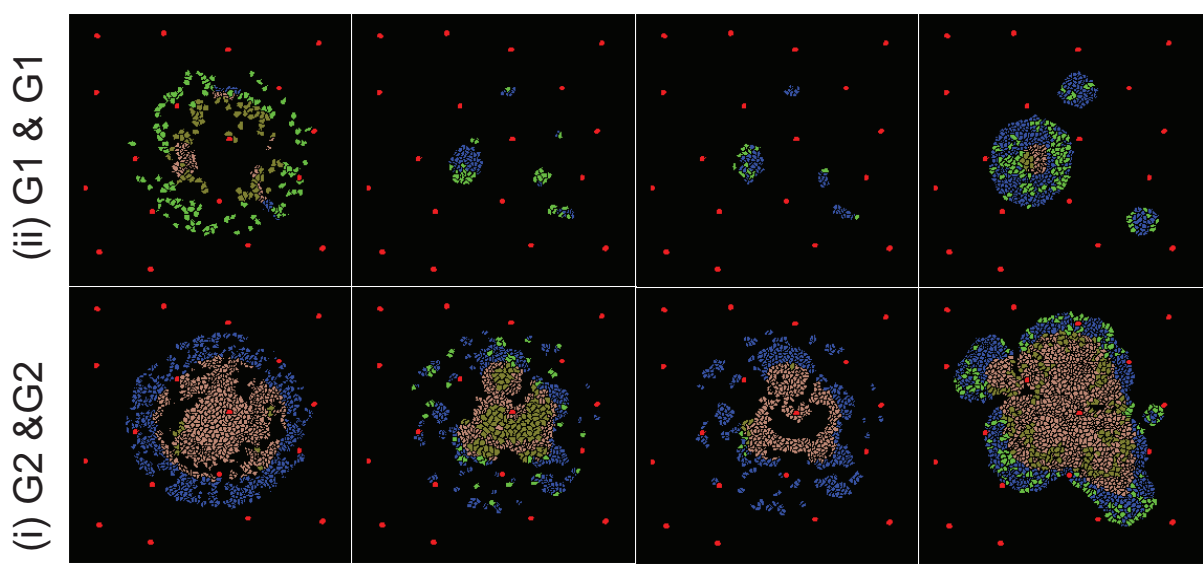

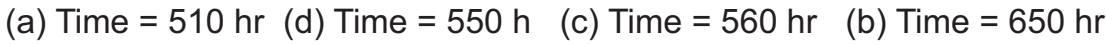

Fig. 5. Plots showing the spatial evolution of tumour cells when cell-cycle phase specific chemotherapeutic drugs are given. (i) G2 drug followed by G2 drug and (ii) G1 drug followed by G1 drug at times (a) $510 \mathrm{hr}$, (b) $550 \mathrm{hr}$, (c) $560 \mathrm{hr}$ and (d) $750 \mathrm{hr}$. Adapted from Powathil et al. ${ }^{6}$.

dynamics. Hence, denoting by $C_{i}(x, t)$ the concentration of chemotherapeutic drug type $i$, its spatio-temporal evolution is given by the equation:

$$
\frac{\partial C_{i}(x, t)}{\partial t}=\nabla \cdot\left(D_{c i}(x) \nabla C_{i}(x, t)\right)+r_{c i}(x) m(x)-\phi_{c i} C_{i}(x, t) \operatorname{cell}(x, t)-\eta_{c i} C_{i}(x, t)
$$

where $D_{c i}(x)$ is the diffusion coefficient of the drug, $\phi_{c i}$ is the uptake rate by a cell (assumed to be zero), $r_{c i}$ is the drug supply rate by the pre-existing vascular network and $\eta_{c i}$ is the drug decay rate ${ }^{5,6}$. As similar to that of equation governing the oxygen concentration, this PDE is incorporated into the CompuCell3D as diffusive chemical field and simulated using the parameters values found in Powathil et al. ${ }^{5}$. To study the effects of multiple phase-specific chemotherapy, we consider two types of phase-specific chemotherapeutic drugs that are either G1 specific or G2-S-M specific, delivered at a same rate. Furthermore, chemotherapeutic drugs are assumed to be effective in killing a cell, if its average concentration at the location of that specific cell is above a fixed threshold value and below which the drug has no effect on any cells. In the following subsections, we study the effects of cell-cycle based chemotherapeutic drugs on a growing tumour using the CompuCell3D framework hybrid multiscale computational model.

\subsection{Homogenous Population Model: The Effects of Chemotherapy}

In this section, we study we effects of cell-cycle based chemotherapy on a population of homogeneously growing tumour cells with similar cell-cycle dynamics (i.e. 


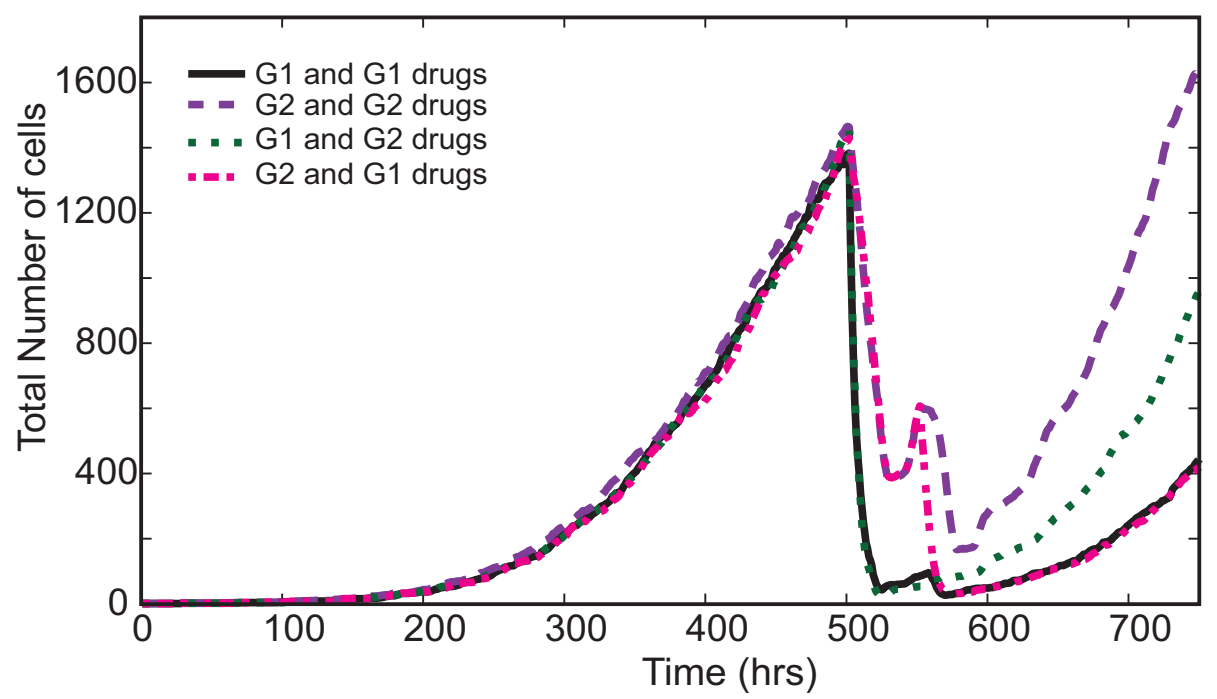

Fig. 6. Plots comparing the total number of cells when the tumour cells are treated with two doses of cell-cycle phase specific drugs at Time $=500 \mathrm{hr}$ and Time $=550 \mathrm{hr}$. Adapted from Powathil et al. ${ }^{6}$.

the same cell-cycle time under favourable conditions) but with intracellular and microenvironmental heterogeneities. Figure 4 shows the spatio-temporal evolution of a solid tumour mass with a homogenous cell population in the absence of chemotherapy. As illustrated in the figure, the colours of tumour cells indicate the cell-cycle position and oxygenation status of each individual cell. The spatial distribution of the tumour cells shows the development of the proliferating rim around the boundary of the growing tumour as the internal cells become hypoxic due to the increased consumption of oxygen supplied from the blood vessels.

To study the effects of cell-cycle, phase-specific chemotherapeutic drugs on the growing tumour, two doses of cell-cycle phase-specific drugs that act on cells that are either in G1-phase or S-G2-M phase are delivered at a same rate at times 500 hours and 550 hours. A representative figure showing the spatio-temporal evolution of cancer cells when the tumour mass is treated with two doses of G1 drugs and G2 drugs is given Figure 5. Figure 6 shows and compares the total number of tumour cells during the therapy. As previously shown by Powathil et al. ${ }^{5}$, the results indicate that the choice and sequencing of different types of chemotherapeutic drugs can significantly affect the spatial distribution and the cytotoxic cell-kill of cancer cells. Furthermore, it has been shown that various factors such as the spatial distribution of cancer cells, the correct sequencing of chemotherapeutic drugs, and intracellular and microenvironment heterogeneities play important roles in determining the precise cytotoxic effectiveness of cell-cycle phase-specific chemotherapeutic drugs.

The results of multiple combinations of cell-cycle specific chemotherapeutic 
November 1, 2014 15:32 Powathil\&Chaplain Manuscript Powathil'BRL'R3

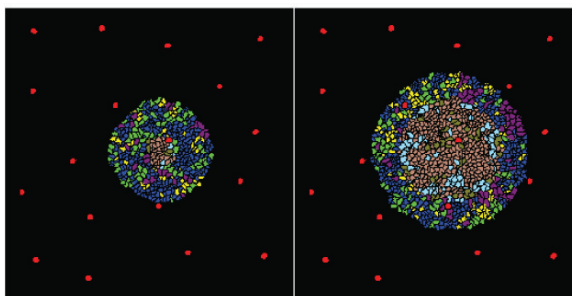

(a) Time $=350 \mathrm{hr}$ (b) Time $=450 \mathrm{hr}$

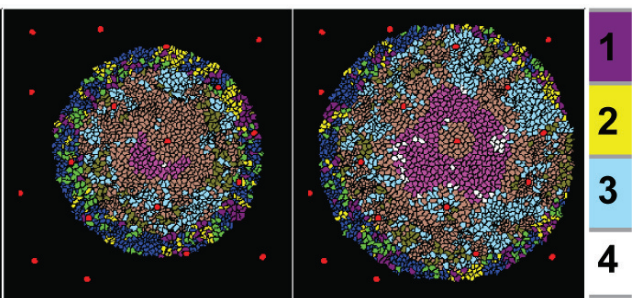

(c) Time $=550 \mathrm{hr} \quad$ (d) Time $=650 \mathrm{hr}$

Fig. 7. Plots showing snapshots of the simulation results of the model with two subpopulations of cancer cells at time points (a) $350 \mathrm{hr}$, (b) $450 \mathrm{hr}$, (c) $550 \mathrm{hr}$ and (d) $650 \mathrm{hr}$. The colour legend given in addition to that of Figure 4 (subpopulation 1) shows the types of the second subpopulation of tumour cells; 1 - G2 phase, 2 - G1 phase, 3 - hypoxic and 4 - resting cells. Adapted from Powathil et al. ${ }^{6}$.

drugs (Figure 6) show that a combination of G1 specific drug followed by another G1 specific drug (Figure 5(ii)) and G2 specific drugs and G1 specific drugs give better therapeutic outcomes than other two combinations. This is due to the presence of a higher fraction of proliferating cells in G1-phase at the time of the drug doses and increased proliferation after the initial dose. However, please note that these drug combinations need not always give the best outcome, especially if there were a higher proportion of resting cells within a growing tumour mass ${ }^{5}$. Hence, it is important to know the underlying spatial distribution of a growing tumour mass and the internal cellular heterogeneities present to achieve the best possible outcome.

\subsection{Heterogeneous Population Model: The Effects of Chemotherapy}

One of the common reasons for chemotherapeutic failure in cancer patients is the emergence of drug resistance in subpopulations within the growing tumour ${ }^{2}$. There are several reasons that contribute to this chemotherapeutic drug resistance, including multi-drug resistance to the chemotherapeutic drugs and the emergence of heterogenous subpopulations with varying responsiveness to the given drug ${ }^{33,34}$. Recently, it has been shown that the tumour heterogeneity caused by the cellcycle dynamics and the variations in the cell-cycle duration can play a vital role in the chemotherapeutic sensitivity, as most of the chemotherapeutic drugs act on actively cycling cells. Several studies involving heterogenous tumour masses that contain a slowly-cycling subpopulation of tumour cells indicated that the use of traditional chemotherapeutic drugs could ultimately lead to an emerging subpopulation of drug resistant, slowly-cycling tumour cells that has the potential to repopulate the tumour mass ${ }^{33,34}$. Moreover, the results from recent computational studies using multiscale mathematical models have also confirmed the role of slowly-cycling tumour subpopulations in developing chemotherapeutic resistance and showed that 

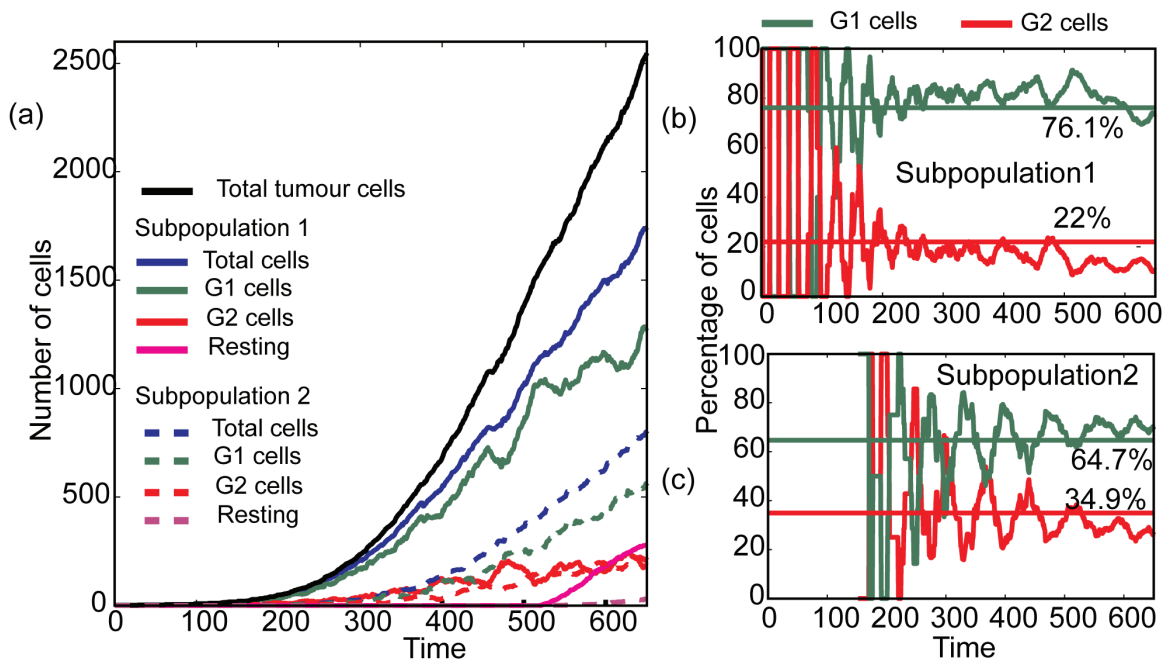

Fig. 8. Plots showing (a) the number of cells in various phases of the cell-cycle for a heterogenous tumour with a second subpopulation of cells of slow-cycling tumour cells, (b) average percentage of cells in G1/G0 and S/G2/M phases for subpopulation 1 and (c) average percentage of cells in G1/G0 and S/G2/M phases for subpopulation 2. The lines represent the corresponding temporal average number of cells either in G1 phase or S-G2-M phases

conventional chemotherapy may sometimes result in the emergence of dominant, slowly-cycling subpopulations of tumour cells ${ }^{6}$.

Recently, Powathil et al. ${ }^{6}$ studied the chemotherapeutic effects of anti-cancer drugs on a tumour mass that consists of two subpopulations: one with an active cell-cycle with a cell-cycle length of 25-30 hours, and a second subpopulation with slowly-cycling tumour cells. Figure 7 shows the spatio-temporal evolution of the heterogenous tumour mass with two subpopulations of tumour cells. The slowlycycling tumour subpopulation is introduced into the previous homogenous model (Figures 4 and 7) through random mutations that are assumed to occur after 100 mcs (hr). The quantitative results of the heterogenous tumour growth model is given in Figure 8. Figure 8(a) compares the total number of tumour cells and the number of cells in various phases of the cell-cycle for both subpopulations 1 and 2 , and Figures $8(\mathrm{~b})$ and $8(\mathrm{c})$ give the percentage of proliferating cells in subpopulations 1 and 2. The results shown in Figures 8(b) and 8(c) indicated that the slow-cycling subpopulation has more cells in G2 phase when compared to subpopulation 1, as observed in previous experimental studies ${ }^{33,34}$.

The heterogenous two population tumour growth model described above can also be used to study the effects of cell-cycle phase-specific chemotherapy ${ }^{6}$. Two doses of cell-cycle phase-specific chemotherapeutic drugs are given at times 500 hours and 550 hours, in a similar manner to that of the homogenous case. A representative figure for the spatial evolution of cancer cells during and after the chemotherapeutic 
November 1, 2014 15:32 Powathil\&Chaplain Manuscript $\quad$ Powathil'BRL`R3

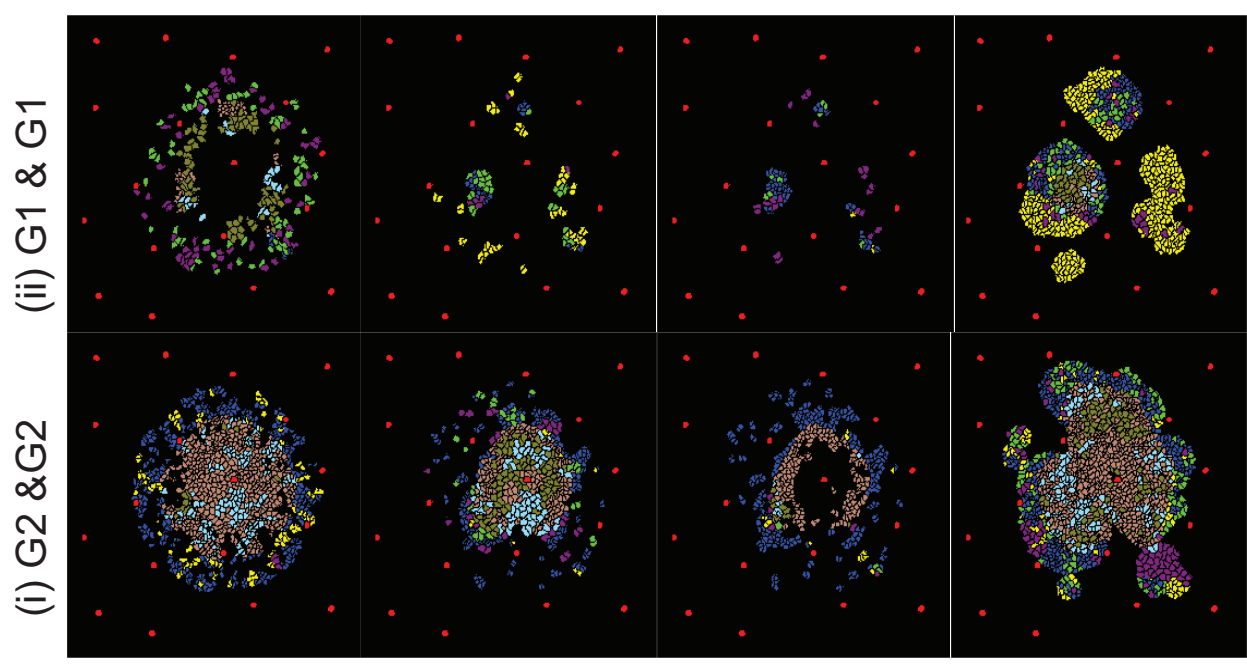

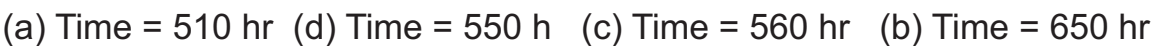

Fig. 9. Plots showing the spatial evolution of tumour cells within a two population model when cell-cycle phase specific chemotherapeutic drugs are given. (i) G2 drug followed by G2 drug and (ii) G1 drug followed by G1 drug at times (a) $510 \mathrm{hr}$, (b) $550 \mathrm{hr}$, (c) $560 \mathrm{hr}$ and (d) $650 \mathrm{hr}$. Adapted from Powathil et al. ${ }^{6}$.

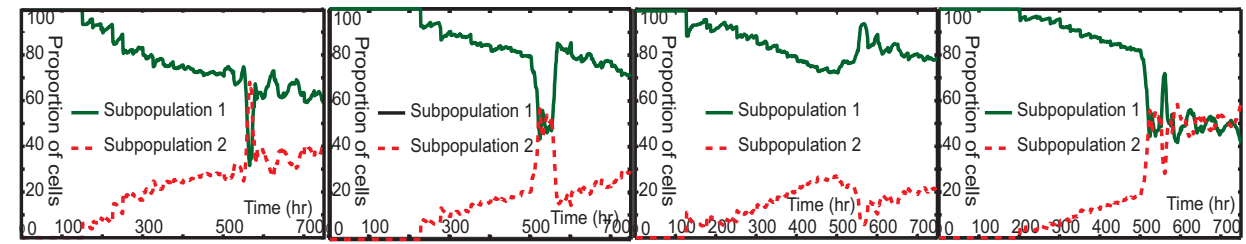
(i) $\mathrm{G} 2 \& \mathrm{G} 1$
(ii) $\mathrm{G} 1 \&$ G2
(iii) $\mathrm{G} 2$ \&G2
(iv) $\mathrm{G} 1 \& \mathrm{G} 1$

Fig. 10. Plots showing percentage of proliferating cells in subpopulations 1 and 2. Adapted from Powathil et al. ${ }^{6}$.

treatment with two doses of G1-phase specific drugs and G2-phase specific drugs is shown in Figure 9. Figure 10 shows the percentage of proliferating cells in subpopulations 1 and 2 when the tumour mass is treated with each combination of cell-cycle phase specific chemotherapeutic drugs. A comparison of the total number of tumour cells and the number of cells in each subpopulations is given in Figure 11 and it shows that combinations of G2 \& G1 specific drugs and G1\& G1 specific drugs give a better outcome than other two combinations. Moreover, it can be seen from Figure 10 that a second dose of G1-phase drug kills a majority of the cancer cells in subpopulations 1 and 2, enriching the slowly-cycling cells in subpopulation 2. These results by Powathil et al. ${ }^{6}$ are in good qualitative agreement with the experimental results of Moore et al. ${ }^{33,34}$. They have shown that when a heterogenous 

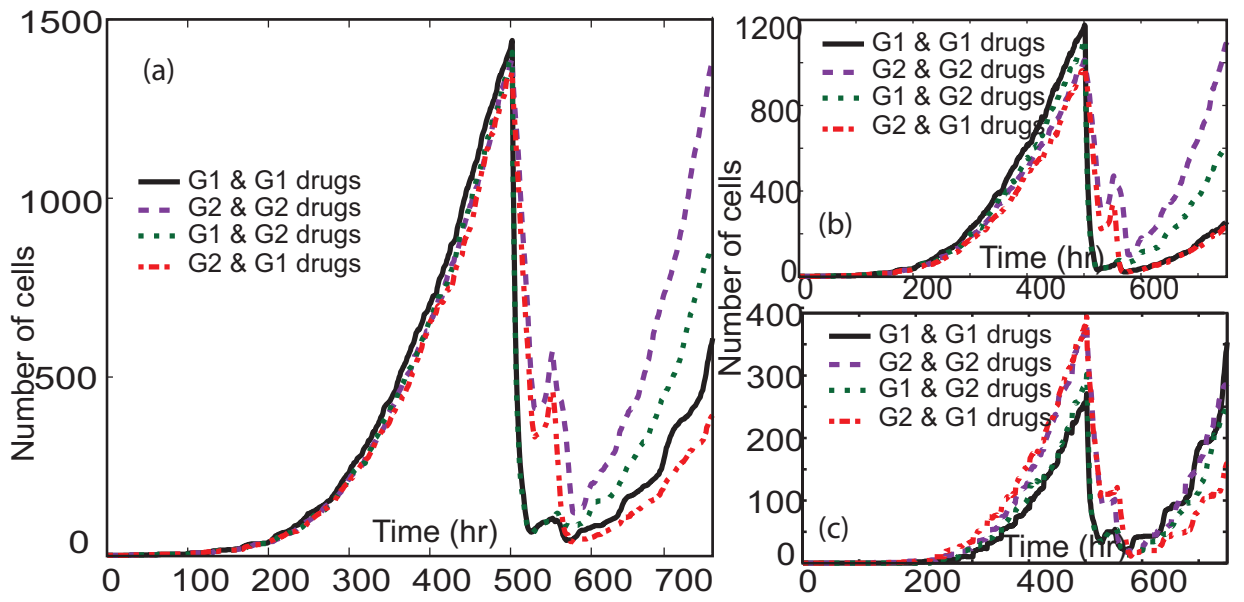

Fig. 11. Plots showing the effects of cell-cycle specific chemotherapeutic drugs on the total number of tumour cells. (a) Total number of cells in two population model, (b) number of cells in subpopulation 1 and (c) number of cells in subpopulation 2. Adapted from Powathil et al. ${ }^{6}$.

tumour mass responds positively to chemotherapeutic treatment, it kills a majority of the active cells, increasing the percentage of slowly-cycling tumour cells within the tumour mass (as shown in Figure 10(iv)) and thus increasing the chance for tumour recurrence. Further extensive analysis and results of the multiscale model for a heterogenous tumour population can be found in Powathil et al. ${ }^{6}$.

\section{Conclusions}

In most cases, chemotherapy is administered as a combination of multiple anticancer drugs that target various processes involved in cancer growth and progression. Combination therapy is usually used to increase the cytotoxicity and mostly targets various intracellular biochemical concentrations that are fluctuating during the cell-cycle, aiming to reduce the drug resistance due to the heterogenous nature of the tumours, with minimal toxicity. However, the efficacy of these administered chemotherapeutic drugs is often influenced by the intracellular perturbations of the cell-cycle dynamics, inducing a cell-cycle-mediated drug resistance. Hence, it is very important to study and analyse the underlying heterogeneity within a cancer cell and within a solid tumour mass due to the presence of the microenvironment and the cell-cycle position so as to design and develop more effective treatment protocols.

In this article, we have presented a multiscale mathematical model incorporating the effects of intracellular cell-cycle dynamics and the external microenvironment to study the spatio-temporal dynamics of tumour growth and its response to cell-cycle based chemotherapy ${ }^{5,6}$. The developed multiscale mathematical model can be implemented using various computational approaches. Here, the multiscale mathematical model is implemented using two hybrid individual-based approaches, namely: (i) a 
November 1, 2014 15:32 Powathil\&Chaplain Manuscript Powathil'BRL'R3

hybrid cellular automaton approach ${ }^{5,9}$ and (ii) a hybrid cellular potts approach using the CompuCell3D framework ${ }^{6}$. Although each of these modelling approach is technically different, the results obtained from both multiscale models are similar and comparable, showing the robustness of the multiscale mathematical modelling approach. Recently, several experimental and clinical observations ${ }^{14,33,34}$ have indicated the role of internal and external heterogeneities in inducing chemotherapeutic drug resistance within a growing tumour, increasing its chances of recurrence. We have further used the multiscale computational model (using the Compucell3D framework) to study the effects and efficacy of chemotherapy on a homogeneously growing tumour with intracellular heterogeneities and a heterogenous tumour growth model (with a slowly-cycling tumour subpopulation) with intracellular heterogeneities. The results obtained from the multiscale model were in very good agreement with the previous experimental findings ${ }^{6,33,34}$ and highlighted the role of intrinsic cell-cycle-driven drug resistance of slowly-cycling tumour subpopulation in the recurrence of the tumour after therapy. Future work will consider other factors that may induce drug resistance within a growing tumour mass such as variations in cell cycle control, anti-apoptotic proteins, multi-drug resistance through the activation of cellular pumps and increased metabolic activities ${ }^{33,35,4}$ to study their role in tumour recurrence and analyse the various optimum delivery protocols for multiple chemotherapeutic drugs to achieve maximum therapeutic benefit.

\section{Acknowledgments}

The authors gratefully acknowledge the support of the ERC Advanced Investigator Grant 227619, M5CGS - From Mutations to Metastases: Multiscale Mathematical Modelling of Cancer Growth and Spread.

\section{References}

1. J. C. Bailar and H. L. Gornik. N. Engl. J. Med. 336, 1569-1574 May (1997).

2. M. A. Shah and G. K. Schwartz. Clin. Cancer Res. 7, 2168-2181 Aug (2001).

3. L. Ding, T. J. Ley, D. E. Larson, C. A. Miller, D. C. Koboldt, J. S. Welch, J. K. Ritchey, M. A. Young, T. Lamprecht, M. D. McLellan, J. F. McMichael, J. W. Wallis, C. Lu, D. Shen, C. C. Harris, D. J. Dooling, R. S. Fulton, L. L. Fulton, K. Chen, H. Schmidt, J. Kalicki-Veizer, V. J. Magrini, L. Cook, S. D. McGrath, T. L. Vickery, M. C. Wendl, S. Heath, M. A. Watson, D. C. Link, M. H. Tomasson, W. D. Shannon, J. E. Payton, S. Kulkarni, P. Westervelt, M. J. Walter, T. A. Graubert, E. R. Mardis, R. K. Wilson, and J. F. DiPersio. Nature 481(7382), 506-510 Jan (2012).

4. V. Koshkin and S. N. Krylov. PLoS ONE 7(7), e41368 (2012).

5. G. G. Powathil, K. E. Gordon, L. A. Hill, and M. A. J. Chaplain. J Theor Biol 308, 1-9 May (2012).

6. G. G. Powathil, M. A. J. Chaplain, and M. Swat. arXiv:1407.0865 [q-bio.TO] (2014).

7. N. Goda, H. E. Ryan, B. Khadivi, W. McNulty, R. C. Rickert, and R. S. Johnson. Mol. Cell. Biol. 23, 359-369 Jan (2003).

8. L. B. Gardner, Q. Li, M. S. Park, W. M. Flanagan, G. L. Semenza, and C. V. Dang. J. Biol. Chem. 276, 7919-7926 Mar (2001). 
November 1, 2014 15:32 Powathil\&Chaplain Manuscript Powathil'BRL'R3

18 MAJ Chaplain and GG Powathil

9. G. G. Powathil, D. J. Adamson, and M. A. J. Chaplain. PLoS Comput. Biol. 9(7), e1003120 Jul (2013).

10. J. H. Goldie and A. J. Coldman. Cancer Treat Rep 63(11-12), 1727-1733 (1979).

11. O. Lavi, M. M. Gottesman, and D. Levy. Drug Resist. Updat. 15(1-2), 90-97 (2012).

12. P. Vaupel. Strahlenther Onkol 166, 377-386 Jun (1990).

13. R Weinberg. The biology of cancer. Garland Science, Taylor and Francis Group, (2006).

14. G. K. Schwartz and M. A. Shah. J. Clin. Oncol. 23, 9408-9421 Dec (2005).

15. R. M. Douglas and G. G. Haddad. J. Appl. Physiol. 94, 2068-2083 May (2003).

16. M. D. Garrett and A. Fattaey. Curr. Opin. Genet. Dev. 9, 104-111 Feb (1999).

17. J. J. Tyson and B. Novak. J. Theor. Biol. 210, 249-263 May (2001).

18. B. Novak and J. J. Tyson. Biochem. Soc. Trans. 31, 1526-1529 Dec (2003).

19. B. Novak and J. J. Tyson. J. Theor. Biol. 230, 563-579 Oct (2004).

20. T. Alarcon, H. M. Byrne, and P. K. Maini. J. Theor. Biol. 229, 395-411 Aug (2004).

21. A. A. Patel, E. T. Gawlinski, S. K. Lemieux, and R. A. Gatenby. J. Theor. Biol. 213, 315-331 Dec (2001).

22. A. Dasu, I. Toma-Dasu, and M. Karlsson. Phys Med Biol 48, 2829-2842 Sep (2003).

23. G. Powathil, M. Kohandel, M. Milosevic, and S. Sivaloganathan. Comput Math Methods Med 2012, 410602 (2012).

24. N. Goda, S. J. Dozier, and R. S. Johnson. Antioxid. Redox Signal. 5, 467-473 Aug (2003).

25. A. R. Kansal, S. Torquato, G. R. Harsh IV, E. A. Chiocca, and T. S. Deisboeck. BioSystems 55, 119-127 Feb (2000).

26. A. R. Anderson and M. A. Chaplain. Bull. Math. Biol. 60, 857-899 Sep (1998).

27. S. Turner and J. A. Sherratt. J. Theor. Biol. 216, 85-100 May (2002).

28. T. Alarcon, H. M. Byrne, and P. K. Maini. J. Theor. Biol. 225, 257-274 Nov (2003).

29. B.T. Ribba, T. Alarcon, K. Marron, P.K Maini, and Z Agur. Lect. notes comput. sci 3305, 444-453 (2004).

30. P. Gerlee and A. R. Anderson. J. Theor. Biol. 246, 583-603 Jun (2007).

31. J. Moreira and A Deutsch. Advances in Complex Systems (ACS) 5(02), 247-267 (2002).

32. V. Andasari, R. T. Roper, M. H. Swat, and M. A. Chaplain. PLoS ONE 7(3), e33726 (2012).

33. N. Moore, J. Houghton, and S. Lyle. Stem Cells Dev. 21(10), 1822-1830 Jul (2012).

34. N. Moore and S. Lyle. J Oncol 2011 (2011).

35. N. Navin, J. Kendall, J. Troge, P. Andrews, L. Rodgers, J. McIndoo, K. Cook, A. Stepansky, D. Levy, D. Esposito, L. Muthuswamy, A. Krasnitz, W. R. McCombie, J. Hicks, and M. Wigler. Nature 472(7341), 90-94 Apr (2011). 